\title{
Fuzzy-Neuro Controler for Smart Fault Detection of a Beam
}

\author{
Harish Ch. Das \\ Department of Mechanical Engineering, I.T.E.R, Bhubaneswar, Orissa, India, 751030
}

\author{
Dayal R. Parhi \\ Department of Mechanical Engineering, N.I.T. Rourkela, Orissa, India, 769008
}

\begin{abstract}
(Received 17 November 2008; accepted 29 April 2009)
This paper addresses the fault detection of a cracked cantilever beam using a hybrid artificial intelligence technique. The hybrid technique used here uses a fuzzy-neuro controller. The fuzzy-neuro controller has two parts. The first part is comprised of the fuzzy controller, and the second part is comprised of the neural controller. The input parameters of the fuzzy controller are relative deviation of the first three natural frequencies and the relative values of the percentage deviation for the first three mode shapes. The output parameters of the fuzzy controller are initial relative crack depth and initial relative crack location. The input parameters of the neural segment of the fuzzy-neurocontroller are relative deviation of the first three natural frequencies and relative values of percentage deviation for the first three mode shapes, along with the initial outputs of the fuzzy controller. The output parameters of the fuzzy-neuro controller are final relative crack depth and final relative crack location. For deriving the fuzzy rules and training patterns of natural frequencies, mode shapes, crack depths and crack locations, theoretical expressions have been developed. Several fuzzy rules and training patterns for the fuzzy controller and neural controller of fuzzy-neuro controller are derived respectively. Experimental set-up has been developed for verifying the robustness of the fuzzy-neuro controller. The results of the developed fuzzy-neuro controller and experimental method are in good agreement.
\end{abstract}

\section{INTRODUCTION}

Scientists throughout the globe are focusing on the behavior of natural frequencies and mode shapes of a vibrating system due to the presence of a crack. Intensive studies are being conducted to find an effective tool for predicting of the location and extent of a crack in a cracked beam.

The method of crack localization and crack size in a beam has been obtained by Karthikeyan et al., ${ }^{1}$ taking the noise error into account from free and forced response. This method has been illustrated through numerical examples. The natural frequencies for bending vibrations of Timoshenko cracked beams with simple boundary conditions have been obtained by Loya et al. ${ }^{2}$, considering beam model as two segments connected by two mass less springs. The free and forced vibration analysis of a cracked beam was performed by Orhan $^{3}$ to identify the crack in a cantilever beam. He has suggested that free vibration analysis provides suitable information for the detection of single and two cracks. Determining the crack position and size in a plate using vibration analysis has been investigated by Higashi et al. ${ }^{4}$ They have proposed an estimation method relating natural frequencies to stiffness of structure for crack identification. Chen et al. ${ }^{5}$ have experimentally identified the crack location and size by plotting curves of the crack-equivalent stiffness versus crack location for the first three natural frequencies, and the intersection of the three curves predicts the crack location and size. Pandey et al. ${ }^{6}$ has shown that changes in the flexibility matrix for lower frequency modes of vibration of a damaged structure can be used for identifying the presence of the damage. Rao et al. ${ }^{7}$ have presented a methodology for structural fault detection using fuzzy logic that is used to determine the health status of a structure. The geometric parameters, as well as the effects of service conditions on the physical and mechanical properties of smart structures, are vaguely understood and therefore cannot be precisely defined as stated by Moens et al. ${ }^{8}$ Parhi $^{9}$ has designed an experimentally validated fuzzy logic controller for mobile robot navigation. Fuzzy rules embedded in the controller enable it to avoid obstacles from mobile robots. Rajakarunakaran et al. ${ }^{10}$ have developed an artificial neural network (ANN) model by using two approaches: a) a feed-forward network with a back-propagation algorithm and b) a binary adaptive resonance network for the fault detection of a centrifugal pumping system. Suresh et al. ${ }^{11}$ have computed modal frequency parameters considering flexural vibration of a transverse cracked cantilever beam at various crack locations and depths that are finally used to train a neural network to identify both the crack location and depth. Sahin et al. ${ }^{12}$ have developed a damage-detection algorithm by using changes in natural frequencies and curvature mode shapes as input in the ANN model to predict damage location and severity in beam structures.

A neural network-based approach has been used by Kao et al. ${ }^{13}$ for detecting structural damage. An experimentally verified damage detection algorithm has been proposed by Huijian et al. ${ }^{14}$, using changes in natural frequencies and strain mode shapes as input to ANN to predict damage in beam-like structures. The structural-damage detection has been proposed by Fang et al. ${ }^{15}$ using frequency response functions (FRFs) as input data to the back-propagation neural network (BPNN). The analysis is used to predict damage severity in cantilever beam. A fuzzy-logic control technique is described by Das et al. ${ }^{16}$ for the prediction of structural damage.

In this paper a new methodology has been proposed for prediction of crack depth and its location using fuzzy-neuro technique. At the beginning, theoretical analyses have been developed to find out the effect of crack depth and crack location 